LA-UR-99-5852

Approved for public release; distribution is unlimited.

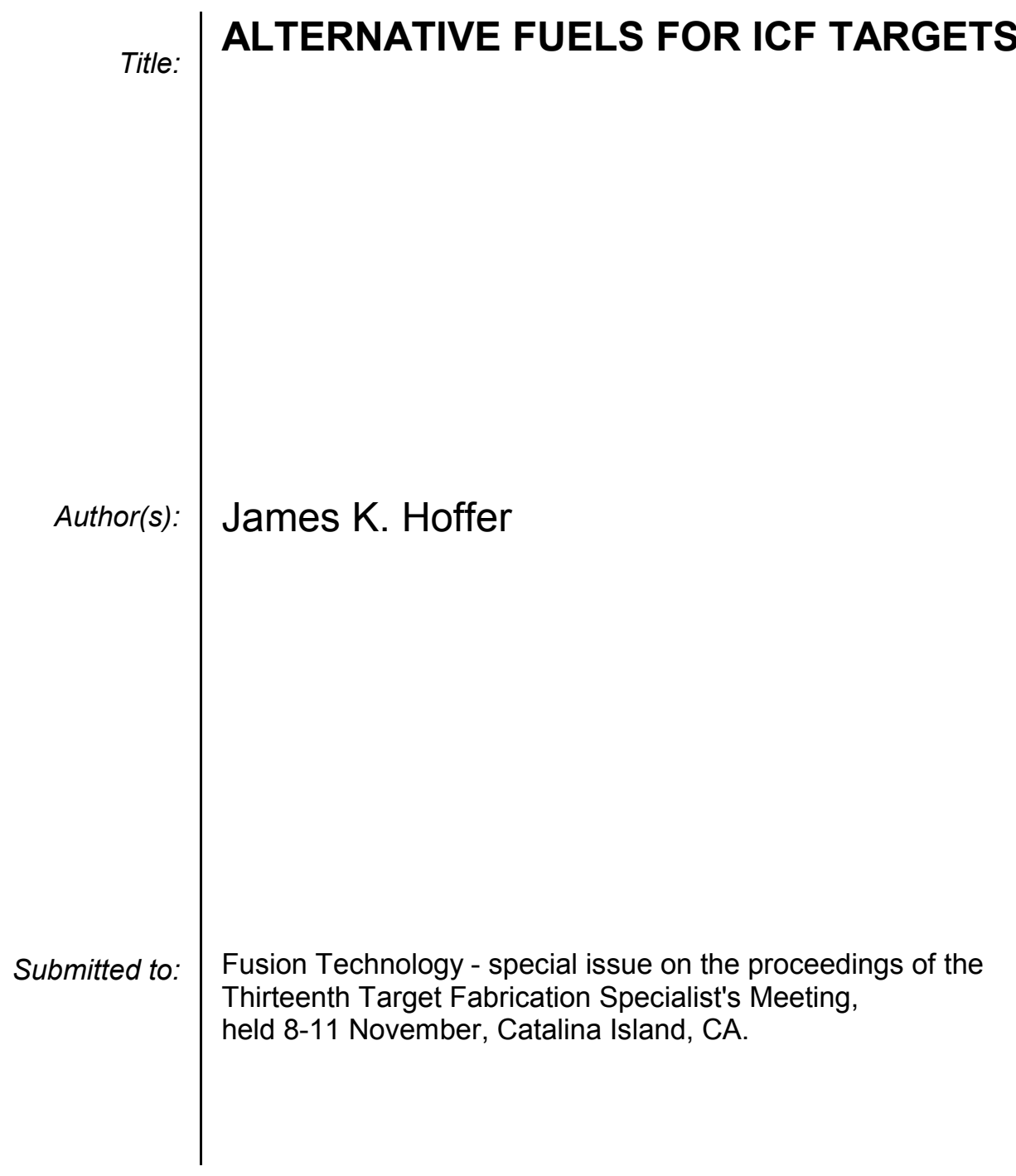

Los Alamos National Laboratory, an affirmative action/equal opportunity employer, is operated by the University of California for the U.S. Department of Energy under contract W-7405-ENG-36. By acceptance of this article, the publisher recognizes that the U.S. Government retains a nonexclusive, royaltyfree license to publish or reproduce the published form of this contribution, or to allow others to do so, for U.S. Government purposes. Los Alamos National Laboratory requests that the publisher identify this article as work performed under the auspices of the U.S. Department of Energy. Los Alamos National Laboratory strongly supports academic freedom and a researcher's right to publish; as an institution, however, the Laboratory does not endorse the viewpoint of a publication or guarantee its technical correctness. 


\title{
ALTERNATIVE FUELS FOR ICF TARGETS
}

\author{
James K. Hoffer \\ Los Alamos National Laboratory \\ Los Alamos, New Mexico 87545 \\ (505) 667-4049
}

\begin{abstract}
The first observation of the beta-layering phenomenon showed that it was possible to fabricate inertial confinement fusion (ICF) targets having an outer ablating shell surrounding a symmetric solid layer of DT fusion fuel. The sensitivity of fusion yield to the internal DT ice roughness is a function of many factors, one of which is the relatively low density of solid DT $\left(0.25 \mathrm{~g} / \mathrm{cm}^{3}\right)$, leading to a high Atwood number for the ablator/fuel interface. This is one of the issues that has led us to consider other DT-based fuels having higher densities than pure DT but still capable of being automatically redistributed into a uniform layer by beta-layering.

The two principle conditions for beta-layering redistribution, self-heating and a moderately high vapor pressure, can be found in only a few other systems. But by concentrating on hydrides of elements in the second row of the periodic chart, we can find materials which should beta-layer and which might be good candidates for fusion fuel. We exclude lithium hydride and beryllium hydride, because these materials are solids at room temperature where an automatic redistribution technique such as betalayering would not be necessary. Therefore we begin with boron and consider the following materials:

- diborane: $\mathrm{B}_{2}(\mathrm{DT})_{3}\left(\right.$ or $\left.\mathrm{B}_{2} \mathrm{D}_{3} \mathrm{~T}_{3}\right)$,

- methane: $\mathrm{C}(\mathrm{DT})_{2}\left(\right.$ or $\left.\mathrm{CD}_{2} \mathrm{~T}_{2}\right)$,

- ammonia: $\mathrm{N}(\mathrm{DT})_{3 / 2}$ (i.e., $\left.0.5 \cdot \mathrm{NDT}_{2}+0.5 \cdot \mathrm{ND}_{2} \mathrm{~T}\right)$,

- water: DT0, and

- hydrofluoric acid: (DT) $)_{1 / 2} \mathrm{~F}$ (i.e., 0.5·DF + 0.5·TF).
\end{abstract}

\section{INTRODUCTION}

The first observation ${ }^{1}$ of the beta-layering phenomenon showed that it was possible to fabricate inertial confinement fusion (ICF) targets having an outer ablating shell surrounding a symmetric solid layer of DT fusion fuel. DT layers prepared by natural beta-layering typically have an internal rms surface roughness ${ }^{2}$ of $\sim 1.3 \mu \mathrm{m}$, permitting good fusion yield within beryllium ablators. However, for a polyimide ablator target, and especially for a $\mathrm{C}-\mathrm{H}$ plastic ablator, the DT surface may need to be smoothed by an enhancement technique such as infrared illumination $^{3}$ or Joule heating ${ }^{4}$. The sensitivity of fusion yield to the internal DT ice roughness is a function of many factors, including feed-in/feed-out instabilities ${ }^{5}$. But the relatively low density of solid DT, $0.25 \mathrm{~g} / \mathrm{cm}^{3}$, results in a high Atwood number for the ablator/fuel interface and is certainly a contributor ${ }^{6}$. This is one of the issues that has led us to consider other DT-based fuels having higher densities than pure DT but still capable of being automatically redistributed into a uniform layer by beta-layering.

Beta-layering occurs in DT because the absorption of energetic beta-particles following tritium beta decay causes self-heating of the solid. A temperature gradient is formed within the fuel layer, being hotter on the inside. A thicker layer will have a higher gradient and a higher internal temperature if the outer ablator is a good thermal conductor. Because the saturated vapor pressure of solid DT is quite high just below the triple point temperature, and because it increases with increasing temperature, a thicker layer tends to sublime. Hence, there is a net transport of material, subliming from thicker portions of the fuel layer and condensing on thinner ones, leading to overall uniformity with an exponential time constant $\tau$ defined as (neglecting ${ }^{3} \mathrm{He}$ ):

$$
\tau=\mathrm{H}_{\mathrm{s}} / \mathrm{Q},
$$

where $\mathrm{H}_{\mathrm{s}}$ is the heat of sublimation $(\mathrm{J} / \mathrm{g})$ and $\mathrm{Q}$ is the rate of heat generation $(\mathrm{W} / \mathrm{g})$ in solid DT. For DT at $19 \mathrm{~K}, \tau=$ 26.73 minutes and is independent of layer thickness. If the ablator/fuel interface is isothermal (to within $\sim 2 \mu \mathrm{K}$ !), the ultimate uniformity of a typical $100-\mu \mathrm{m}$-thick fuel layer is better than $0.1 \mu \mathrm{m}$ for any single mode, with a residual surface roughness of $1.3 \pm 0.3 \mu \mathrm{m}$ rms caused mainly by the tendency of hydrogen crystals to form flat facets.

Two principle conditions for beta-layering redistribution are self-heating and a moderately high vapor pressure. Most radioactive elements are solid heavy refractory metals at room temperature and have virtually no vapor pressure at the triple point temperature. Hence, most radioactive materials cannot readily redistribute solid via the vapor phase. 


\section{MATERIALS OF INTEREST}

By concentrating on hydrides of elements in the second row of the periodic chart, we can find materials that should beta-layer and that might be good candidates for fusion fuel. We'll exclude lithium hydride and beryllium hydride, because these materials are solids at room temperature where an automatic redistribution technique such as beta-layering would not be necessary. Therefore we begin with boron and consider the following materials:

- diborane: $\quad \mathrm{B}_{2}(\mathrm{DT})_{3}\left(\right.$ or $\left.\mathrm{B}_{2} \mathrm{D}_{3} \mathrm{~T}_{3}\right)$,

- methane: $\quad \mathrm{C}(\mathrm{DT})_{2}\left(\right.$ or $\left.\mathrm{CD}_{2} \mathrm{~T}_{2}\right)$,

- ammonia: $\mathrm{N}(\mathrm{DT})_{3 / 2}$ (i.e., $0.5 \cdot \mathrm{NDT}_{2}+0.5 \cdot \mathrm{ND}_{2} \mathrm{~T}$ ),

- water: DT0, and

- hydrofluoric acid: (DT) $)_{1 / 2} \mathrm{~F}$ (i.e., 0.5 $\left.\mathrm{DF}+0.5 \cdot \mathrm{TF}\right)$.
Note that we have made the assumption that deuterium and tritium in a 50/50 ratio have replaced all the hydrogen (protium) atoms in the parent material. Let us use the term $\mathrm{X}_{\mathrm{m}}(\mathrm{DT})_{\mathrm{n}}$ or simply XDT to refer to all of the new fuels, i.e., all but pure DT. All quantities listed in table I. refer to the XDT material in the solid state at the triple point temperature $\left(\mathrm{T}_{t}\right)$. In deriving the physical values of the XDT solid phases, it has been assumed that the molar density of each XDT solid is the same as the original (protiated) material. The specific density was then calculated from the molecular weight. Also, except for pure DT, the values listed for $T_{t}$, $\mathrm{H}_{\mathrm{s}}$, and the saturated vapor pressure $\mathrm{P}_{\mathrm{sat}}$ are simply those for the protiated materials ${ }^{7}$. The self-heating is due only to the tritium and therefore the rate of self-heating $\mathrm{Q}$ can be computed exactly for each XDT species.

\begin{tabular}{|c|c|c|c|c|c|c|c|c|c|c|}
\hline Material & Formula & $\begin{array}{l}T_{t} \\
(K)\end{array}$ & $\begin{array}{l}\text { Mol. Wt. } \\
\text { (g/mole) }\end{array}$ & $\begin{array}{c}\mathbf{r} \\
\left(\mathrm{g} / \mathrm{cm}^{3}\right)\end{array}$ & $\begin{array}{l}\text { Mol. Density } \\
\left(\mathrm{mole}\left[\mathrm{DT} / \mathrm{cm}^{3}\right)\right.\end{array}$ & wt. \% DT & $\begin{array}{c}\mathbf{Q} \\
(\mathrm{W} / \mathrm{mole})\end{array}$ & $\begin{array}{c}\mathbf{H}_{\mathrm{s}} \\
(\mathrm{J} / \mathrm{mole})\end{array}$ & $\begin{array}{c}\mathbf{t} \\
\text { (minutes) }\end{array}$ & $\begin{array}{l}\mathbf{P}_{\text {sat }} \\
\text { (torr) }\end{array}$ \\
\hline hydrogen & DT & 19.8 & 5.03 & 0.25 & 0.050 & $100.0 \%$ & 0.977 & 1580 & 27 & 150.7 \\
\hline diborane & $\mathrm{B}_{2} \mathrm{D}_{3} \mathrm{~T}_{3}$ & 108 & 36.71 & 0.77 & 0.063 & $41.1 \%$ & 2.931 & 23000 & 131 & 0.6 \\
\hline methane & $\mathrm{CD}_{2} \mathrm{~T}_{2}$ & 89 & 22.07 & 0.68 & 0.061 & $45.6 \%$ & 1.954 & 9124 & 78 & 75.0 \\
\hline ammonia & $\begin{array}{c}\mathrm{N}(\mathrm{DT})_{3 / 2} \\
5 \mathrm{NDT}_{2}+.5 \mathrm{ND}_{2} \mathrm{~T}\end{array}$ & 195 & 21.55 & 1.03 & 0.072 & $35.0 \%$ & 1.466 & 26575 & 302 & 45.2 \\
\hline water & DTO & 273 & 21.03 & 1.07 & 0.051 & $23.9 \%$ & 0.977 & 50936 & 869 & 4.6 \\
\hline $\begin{array}{l}\text { hydrofluoric } \\
\text { acid }\end{array}$ & $\begin{array}{l}(\mathrm{DT})_{1 / 2} \mathrm{~F} \\
(.5 \mathrm{DF}+.5 \mathrm{TF})\end{array}$ & 181 & 21.51 & 1.65 & 0.038 & $11.7 \%$ & 0.489 & [157000] & [5400] & 1.5 \\
\hline
\end{tabular}

$$
\begin{array}{lll}
\text { notes: } & T_{t}=\text { triple point temperature } & H_{s}=\text { heat of sublimation } \\
& Q=\text { rate of self heating } & t=\text { time constant for beta-layering }=H_{s} / Q
\end{array}
$$

Table I. Physical and thermodynamic properties of alternative fuel materials [estimated].

A few general observations concerning the XDT solid materials are in order. First, they are all considerably denser than pure solid DT. The lowest density is exhibited by methane, but even that is 2.72 times that of pure DT. All of these fuels would be a better density match to the ablator shell (either $\mathrm{Be}$ or $\mathrm{C}-\mathrm{H}$ polymer) than pure DT. We notice that the XDT solids are more efficient than pure DT solid in the packing density for the DT payload, i.e., the molar density related to a mole of DT is greater (except for hydrofluoric acid). This comes at the expense of a lower wt. \% DT, requiring more energy to reach fusion conditions because the higher- $\mathrm{Z} \mathrm{X}$-atoms also will be heated (and will not take part in fusion). The highest value of relative DT density occurs in ammonia where it is $44 \%$ greater than in pure DT. Whether the higher density would offset the energy wasted in heating the nitrogen atoms to fusion ignition temperatures will not be considered here, nor will any possible enhancement of fusion burn due to the higher alpha-particle stopping power of the nitrogen atoms. 


\section{CHEMISTRY}

The protiated species XHH are stable materials except for diborane, $\mathrm{B}_{2} \mathrm{H}_{6}$, which as a gas at room temperature and above tends to decompose to the elemental species, first producing hydrogen gas and higher borane species such as $\mathrm{B}_{4} \mathrm{H}_{10}, \mathrm{~B}_{5} \mathrm{H}_{9}$, etc. However, all the XDT materials considered here would all be unstable due to the tritium beta-activity. For instance, it is known that $\mathrm{CD}_{2} \mathrm{~T}_{2}$ self-polymerizes, forming species such as ethane, $\mathrm{C}_{2} \mathrm{D}_{3} \mathrm{~T}_{3}$, propane, $\mathrm{C}_{3} \mathrm{D}_{4} \mathrm{~T}_{4}$, etc. and DT gas. Ultimately, amorphous $(-\mathrm{CDT}-)_{\mathrm{x}}$ polymers and pure carbon form and plate out on the container ${ }^{8}$. Ammonia would not be expected to polymerize, but will also tend to decompose to its elements.

Except for water, all the XDT materials are chemically reactive. Diborane, methane and ammonia are gases at room temperature and are flammable (although ammonia is a "negligible fire hazard"). Both water and anhydrous hydrofluoric acid are liquids at room temperature (HF boils at $19.4{ }^{\circ} \mathrm{C}$, hence DF and TF probably would have boiling points just above the nominal 'room temperature' of $25^{\circ} \mathrm{C}$ ).

Biologically, DTO is extremely hazardous, because the radioactive tritium is readily exchanged with protium in body tissue. Because of their more active chemistries, all the other XDT materials would be even more hazardous. None of these materials offer any advantages to worker safety and, like DTO, all would be considered to be at least 25,000 times more hazardous than DT gas.

\section{BETA-LAYERING OF PURE XDT MATERIALS}

When beta-layering with fresh DT, the beta-layering time constant $\tau$ is only 27 minutes and the optimum interior surface layer is achieved after roughly 10 time constants, i.e., five hours. Diborane would not be the best candidate for beta-layering because the heat of sublimation is so high that $\tau$ would be over two hours. Moreover, the vapor pressure of diborane at the triple point is so low that the presence of small amounts of ${ }^{3} \mathrm{He}$ (due to just a few hours of aging) or pure DT gas (due to chemical decomposition) would induce substantial increases in $\tau$. Likewise, neither water nor anhydrous hydrofluoric acid appears to be a good candidate, unless one could show that the advantage of near room temperature handling afforded by DTO offsets the expected very slow equilibration time. We note that because we are dealing with the solid phase when we consider beta-layering, even DTO must be kept below normal room temperature.

Of the two remaining, ammonia offers the advantage of higher solid density and slightly higher DT density. However, solid ammonia might be so slow to beta-layer that considerable amounts of $\mathrm{N}_{2}$ and DT gases, as well as the ${ }^{3} \mathrm{He}$ gas resulting from beta-decay, would form and be present as bubbles in the solid, perhaps leading to increased surface roughness at the fuel/vapor interface and/or density defects in the solid fuel. Methane has the advantages of a shorter time constant and a higher vapor pressure but might still suffer from decomposition or polymerization induced sediments produced within the fuel. Nevertheless, the prospect of target handling at temperatures just above that of liquid nitrogen for methane or at dry ice temperature* for ammonia is sufficiently tantalizing that further investigations of either of these two materials, including chemical preparation and cryogenic beta-layering experimentation, is worth considering.

Notwithstanding the tendencies for polymerization or decomposition, both of these latter two materials should freeze as transparent, crystalline solids. Whether the interior roughness of the beta-layered solids would be similar to that observed in solid DT would depend on the exact nature of properties such as crystal morphology or crystalline surface energies ${ }^{9}$ and would be best answered experimentally. However, surface enhancement techniques ${ }^{3,4}$ would be applicable if these materials are sufficiently transparent.

\section{DOUBLE-LAYERED TARGETS}

Consider now the effect of having an excess amount of pure DT in the initial charge of fuel, i.e., a mixture of XDT and pure DT. Beta-layering would have to take place in two stages. First, just below the triple point temperature of the main fuel, the temperature would be held constant until the XDT fuel achieves sufficient symmetry. Then the capsule would be cooled to $\sim 19 \mathrm{~K}$ where the remaining pure DT fuel would form a second inner layer. This "double-layered target" concept could be quite attractive. First, an innermost pure DT layer would enhance the establishment of a 'hotspot' and fusion ignition ${ }^{6}$, as has already been calculated for both Be and C-H capsules containing pure DT fuel. Secondly, the presence of an excess of pure DT would tend to stabilize the XDT species, or at least slow the decomposition processes. Thirdly, the XDT fuel layer would form an intermediate density layer between an outer Be ablator shell and the inner pure solid DT layer. Either methane or diborane would act as an intermediate density layer between an outer container shell of C-H polymer and the inner pure DT layer. The reduced Atwood number at the interfaces should lessen the shock reflection and the destabilizing effects of the "feed-in feed-out" processes ${ }^{5}$, thus promoting ignition.

Can the amount of excess pure DT be too great? The presence of excess DT gas during the layering of the XDT fuel would slow down the beta-layering rate, just as ${ }^{3} \mathrm{He}$ slows down beta-layering ${ }^{10}$ for pure DT. The redistribution

\footnotetext{
${ }^{*}$ We expect the actual triple point temperature of $\mathrm{N}(\mathrm{DT})_{3 / 2}$ to be $\sim 200 \mathrm{~K}$. Therefore it should be frozen at the sublimation temperature of $\mathrm{CO}_{2}(195 \mathrm{~K} @ 1 \mathrm{~atm}$.$) .$
} 
of solid fuel by beta-layering occurs via the gas phase. When an interior gas species does not take part in the sublimation-condensation of the solid species, then it acts as a barrier to the mass transport. The main fuel must then diffuse through the stagnant central gas. Quite small amounts of ${ }^{3} \mathrm{He}$ have strong effects on the beta-layering rate constant. Likewise, if one desired to have a doublelayered target where the inner pure DT layer thickness were any greater than a small percentage of the total fuel charge, then the beta-layering of the XDT layer might take days instead of hours.

\section{THE SELF-ASSEMBLING TARGET}

Consider an outer capsule shell made from a few microns of C-H polymer. Now diffuse into this shell a charge of XDT mixed with a large (50\% to $100 \%)$ excess amount of pure DT. In spite of our caveat above concerning too much excess DT, the first beta-layering might not be so slow after all. The reason for this is subtle. In beta-layering with pure DT and excess ${ }^{3} \mathrm{He}$, the gas density in the center of the capsule is low. Therefore, the temperature across the central vapor space is relatively flat. Whether it is achieved by induced Joule heating or by adsorbed IR illumination, an enhancement of betalayering occurs because a stronger temperature gradient is established in the solid fuel layer. (Joule heating accomplishes this by heating only the interior gas, while IR illumination heats the solid directly.) But during the first beta-layering of the double-layered target, there is already present a source of internal heating in the vapor space: radioactive DT gas! This could more than make up for the stagnant nature of the DT gas and permit rapid formation of the first XDT layer.

One would need to have a high pressure environment for the first beta-layering, due to the high pressure of the interior DT gas at the triple point temperature of the XDT material and the low strength of the outer shell. A spherical permeation vessel could provide this.

If the first XDT layer were satisfactory, then the target could be cooled to $\sim 19 \mathrm{~K}$ where 'normal' betalayering of the pure DT layer would occur. What we would now have is an ideal indirect-drive target - i.e., an outer symmetric ablator of low-Z (XDT) and an inner symmetric layer of pure DT. Except for the outer 'gas bag,' the assembly of these shells both occur automatically! The extra 'expense' of preparing the XDT might well be offset by the convenience of this self-assembling target.

We can go a step further and ask if the use of XHH as the ablator would not also permit a self-assembling target. For example, normal methane, $\mathrm{CH}_{4}$, might betalayer just as effectively as $\mathrm{CD}_{2} \mathrm{~T}_{2}$ ! This is because the presence of the "excess" DT can serve as the driver for symmetrization of the solid $\mathrm{CH}_{4}$ during the first layering.

\section{FRACTIONATION}

Although we use the term DT throughout, we use this as shorthand for the true $50 / 50 \mathrm{D} / \mathrm{T}$ mixture, having relative amounts of molecular species: $0.25 \cdot \mathrm{D}_{2}, 0.5 \cdot \mathrm{DT}$, and $0.25 \cdot \mathrm{T}_{2}$. While effects of fractionation of these different molecular species has been observed between co-existing liquid and gas phases, no effect has been observed in beta-layering of solid DT. During a very slow freeze of liquid DT inside a capsule, we should expect that the first material to freeze out on the shell wall is the highest melting point $\mathrm{T}_{2}$ species, followed by DT, with relatively pure $\mathrm{D}_{2}$ finally forming the last interior layer. However, any such inter-species layering might be quickly washed out by the relatively high value of the self-diffusion constant known to occur in the solid hydrogen. If, as is usually the case, the initial freeze did not occur symmetrically, then the subsequent mass transport via beta-layering would further mix the species. A good example of the lack of fractionation effects in pure DT is the relatively small range over which freezing is observed to occur, i.e., typically just a few $\mathrm{mK}$, whereas the melting points of $\mathrm{D}_{2}$ and $\mathrm{T}_{2}$ differ by almost $2000 \mathrm{mK}$. In effect, freezing DT behaves very much like a eutectic mixture.

However, the degree of fractionation that might occur in the XDT materials has not been determined. For example, at equilibrium, $\mathrm{CD}_{2} \mathrm{~T}_{2}$ should be a mixture of the following molecular species: $0.0625 \cdot \mathrm{CD}_{4}, 0.25 \cdot \mathrm{CD}_{3} \mathrm{~T}, 0.375 \cdot \mathrm{CD}_{2} \mathrm{~T}_{2}$, $0.25 \cdot \mathrm{CDT}_{3}$, and $0.0625 \cdot \mathrm{CT}_{4}$. During the beta-layering of $\mathrm{CD}_{2} \mathrm{~T}_{2}$, (if done slowly) layers of differing composition might result and remain. This would actually be advantageous to the implosion physics, because the outer portion of the fuel layer would be tritium-rich and hence denser while the inner portion would be deuterium-rich. This change in the density of the layer is in the correct direction to reduce Atwood numbers and thus act as a gradual density-matching medium between the outer ablator and the pure DT 'spark plug.'

\section{ACKNOWLEDGEMENTS}

The author is indebted to Robert E. Ellefson and Dean H. Carstens for discussions on materials preparation chemistry. This work was performed under the auspices of the $\mathrm{U}$. S. Department of Energy by the Los Alamos National Laboratory under contract No. W-7405-Eng-36.

\section{DEDICATION}

This work is dedicated to the lasting memory of our colleague Larry R. Foreman, with whom these topics were first developed in April 1992. 


\section{REFERENCES}

1. J. K. Hoffer and L. R. Foreman, Phys. Rev, Lett. 60, 1301 (1988).

2 J. D. Sheliak and J. K. Hoffer, Fusion Technology 35, 234 (1999).

3 D. N. Bittner, G. W. Collins, E. Monsler, and S. Letts, Fusion Technology 35, 245 (1999).

4 E. R. Mapoles, and J. Sater, J. Pipes, and E. Monsler, Phys Rev E. 55, 3473 (1997).

5. T. R. Dittrich, S. W. Haan, M. M. Marinak, S. M. Pollaine, D. E. Hinkel, D. H. Munro, C. P. Verdon, G. L. Strobel, R. McEachern, R. C. Cook, C. C. Roberts, D. C. Wilson, P. A. Bradley, and L. R. Foreman, Physics of Plasmas 6, 2164 (1999);

D. C. Wilson, P.A. Bradley, N.M. Hoffman, F. J. Swenson, D.P. Smitherman, R.E. Chrien, R. W. Margevicius, D.J. Toma, L. R. Foreman, J. K. Hoffer, S. R. Goldman, S. E. Caldwell, T. R. Dittrich, S. W.Haan, M. M. Marinak, S. M. Pollaine, and J.J. Sanchez, Physics of Plasmas 5, 1953 (1998).

6 John Lindl, Physics of Plasmas 2, 3933 (1995).

7. For most of the materials, the triple point temperatures and the solid densities and vapor pressures at the triple points were derived from data found in The Handbook of Chemistry and Physics. Additional vapor pressure data and most heats of sublimation were obtained from the Gmelin Handbook of Inorganic and Organometallic Chemistry and references therein.

8. G. T. McConville and D. A. Menke, Fusion Technology 23, 316 (1993).

9. B. J. Kozioziemski, G. W. Collins, and T. P Bernat, Fusion Technology 31, 482 (1997).

10. T. P. Bernat, E. R. Mapoles, and J. J. Sanchez, Lawrence Livermore National Laboratory Inertial Confinement Fusion Quarterly Report, 1, 57 (1991), UCRL-LR-105821-91-2. 